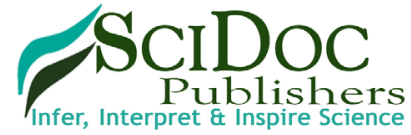

\section{Role of Patellar Imaging using 3D Computed Tomography in Determination of Sex and Stature in Upper Egypt Population}

\author{
Abdelaleem SA ${ }^{1 *}$, Hassan $\mathrm{OA}^{1}$, Abdelazeem IK ${ }^{1}$, Abdel Kader $\mathrm{M}^{2}$
}

${ }^{1}$ Assistant Professor of Forensic Medicine and Toxicology, Faculty of Medicine, Minia University, Egypt.

${ }^{2}$ Assistant Professor of Radiodiagnosis, Faculty of Medicine, Minia University, Egypt.

Abstract

Objective: Patella has been studied for the purpose of sex determination in many cadaveric studies. Since several studies have been demonstrated that discriminant function and linear regression equations used to determine the sex and stature of a skeleton are population specific, the purpose of this study was to evaluate the capability of computed tomography of patella of living subjects to identify sex and stature in Egyptians.

Subjects and Methods: Seven measurements were taken in this study. Data were analyzed by SPSS version 16. All measurements showed significant sexual differences $(\mathrm{P}<0.05)$. Maximal thickness gave a highest percentage of accuracy in sex determination $(85.9 \%)$. By linear regression analysis, the most accurate variable that can predict stature in males was maximal height $(\mathrm{R}=0.933)$ followed by maximal thickness $(\mathrm{R}=0.915)$. Maximal height can also accurately predict stature in females $(\mathrm{R}=0.965)$ followed by maximal breadth of patella $(\mathrm{R}=0.942)$.

Conclusion: Finally, it is concluded that patella of Egyptian population is a useful tool for sex and stature identification.

Keywords: Patella; Sex Determination; Computed Tomography; Discriminant Function; Stature; Linear Regression.

\section{Introduction}

Sex and stature determination using human skeletal parts are very important in many anthropological cases and traumatic events (e.g mass disaster, murder, road traffic accidents). Identification of the individual either living or dead is required in cases of civil \& criminal matters [1]. Sex determination is the $1^{\text {st }}$ and essential step taken by any forensic anthropologist in forensic identification of skeletal remains. Sex identification process has two methods for measurement of data collection namely morphologic and metric methods [2].

The morphological method depends on visual inspection and observation of the sexual differences \& physical characteristics of bones. On the other hand, the metric method was based on measurements \& statistical techniques. This method is used to identify sex from bones that are not so obviously morphologically distinct (e.g small bones of foot, patella). Metric measurements were preferred due to their easy repeatability, high accuracy \& no requirement for special skill [3].

Stature is one of the most important parameters in identification of an individual, living or dead. It can be determined from a skeleton even many years after death. Assessment of height of an individual from measurements of different parts of the body has always been of a great interest and has been done by many anthropologists and forensic experts with variable degree of precision $[4,5]$.

Many bones e.g pelvis, skull \& long bones were used in sex \& stature determination. Recently, one of the skeletal elements drawing more attention is the patella. Patella is a small sesamoid compact bone that is situated in front of the knee joint in the tendon of quadriceps femoris. It is triangular in shape and has 2 surfaces

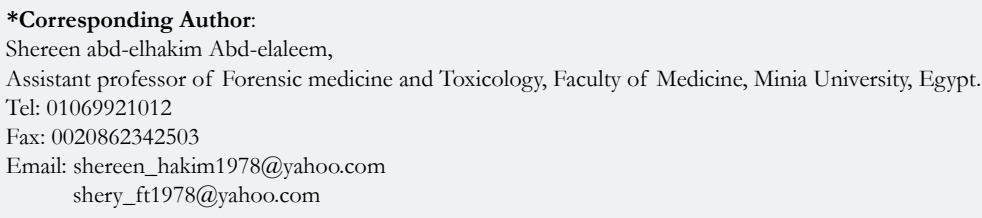

Copyright: Abdelaleem SA ${ }^{\circ}$ 2016. This is an open-access article distributed under the terms of the Creative Commons Attribution License, which permits unrestricted use, distribution and reproduction in any medium, provided the original author and source are credited. 
(anterior \& posterior), three borders and an apex [3].

The patella (knee cap) is considered a solid element of the human skeleton with no obvious morphological features for determining sex or stature. Patella is one of the durable bones that is resistant to postmortem changes \& so it can be available for personal identification. Being a sesamoid bone that is present within the tendon of quadriceps muscle, it was preserved in corpses destroyed by fire [6].

Advances in techniques such as computed tomography (CT scanning), Magnetic resonance image (MRI), computer based anthropometry are answering questions that could not have been answered 10 years ago, and are significantly improving the accuracy of skeletal analysis especially in sex and stature determination [7].

Patellar measurements have been evaluated in prior studies on cadavers for sex determination. Therefore, the aim of this study was to determine the sex \& stature in livings using a noninvasive method which is 3D CT scanning \& develop formulae through utilizing discriminant function \& regression analysis among sample of Egyptian population.

\section{Subjects and Methods}

\section{Subjects}

This study was carried out in Minia University hospital- Egypt on 198 patients (120 male with age ranged from 30-60 \& 78 females with age ranged from 28-63). These patients were recruited from Radiology department and had been subjected to 3D CT for their knees for reasons other than patellar pathologies (e.g patellar osteoarthritis, rheumatoid arthritis, chondromalacia patellae, congenital deformity of patella and previous patellar trauma or surgery. A full informed consent was obtained from all subjects participated in this study. This study has been approved by the human ethical committee council of our university. All the work performed in this study has been carried out in accordance to the code of ethics of the world medical association (declaration of Helsinki). Stature should be measured in all subjects included in this study.

\section{Multidetector CT (MDCT) Protocol for Image Acquisition}

All the patients were in supine position with the patella facing to- wards the ceiling in the extended knee position ( 0 degree of flexion). All patients underwent multi-slice computed tomography using 16- slice MDCT machine (GE bright speed, GE health care, Waukesha, WI, USA). Examinations were done in Minia University hospital- Egypt with the same examination protocol using $64 \times 0.5 \mathrm{~mm}$ Collimation scanner with a gantry rotation speed of $400 \mathrm{~ms} /$ rotation, range of box $450-500$, image thickness $0.5 \mathrm{~mm}$, standard pitch factor of 0.641 , reconstruction interval $0.5 \mathrm{~mm}$ and a total exposure time 6.949. Each scan was obtained with a tube voltage of $120 \mathrm{KV}$ and $250 \mathrm{mAs}$. Images were transferred to a separate advanced workstation (GE, AW 4.4) with commercially available software beside the main apparatus that allows measurements.

\section{Measurements}

Axial cuts taken on the knee region with the above parameters and cut thickness with narrow interval and then reconstructed coronal and sagittal images were generated as well as the $3 \mathrm{D}$ reformatted images that were used to obtain the following measurements. These six measurements have been modified from Knuxmann [8] as follow (Figure 1):

- Maximum height of patella $(\mathrm{MH})$ from the posterior aspect: it is the maximum linear distance from the tip of patellar apex down to the base.

- Maximum facet breadth of patella (MFB): it is the maximum linear distance between the medial \& lateral borders of patella.

- Maximum thickness (MT) on sagittal reconstructed 3D reformatted images: it is the maximum linear distance between the anterior and posterior patellar surfaces.

- Maximum height of articulating facet (MFH): it is the maximum linear distance between the most superior and the most inferior points on the articular facet on the posterior surface.

- External articulating facet width (EFW) (posterior aspect): it is the linear distance between the lateral border of patella and the median ridge of the articulating facet.

- Internal articulating facet width (IFW) (posterior aspect): it is the linear distance between the medial border of the patella median ridge of the articulating facet.

All measurements were taken on the right side by the same radiologist. All 6 measurements were repeated on 20 (10 male \& 10

Figure 1. Photograph of 3D- MSCT of Patella Shows Maximum Height (MH), Maximum Breadth (MFB), Maximum Thickness (MT), Maximum Height of Articulating Facet (MFH), External Articulating Facet Width (EFW), and Internal Articulating Facet Width (IFW).

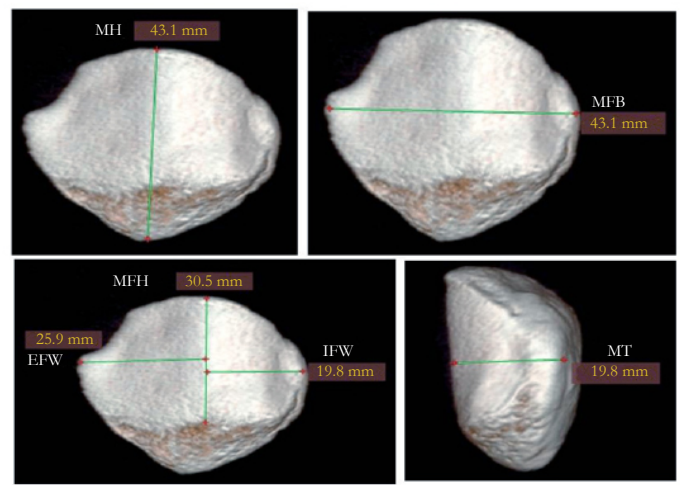


female) patellae of the patients to assess the inter- and intraobserver error \& repeatability. The repeatability of the technique was evaluated using concordance coefficient of reproducibility [9].

\section{Statistical Analysis}

The data were analyzed using SPSS statistical Package version 16. Mean and standard deviations were obtained for stature \& all 6 measurements of patella. Correlation between stature $\&$ different patella measurements was tested using Pearson's correlation test. After using a student- $t$ - test to establish that a significant difference exists $(\mathrm{P}<0.05)$ between male $\&$ female stature $\&$ patellar measurements, discriminant function $\&$ linear regression analyses were performed.

\section{Results}

The results of repeatability test showed that the range of values for the concordance correlation coefficient of reproducibility obtained in this study fell within the internationally standard of 0.90- 0.99 as suggested by Cameron [10] (Table 1). This indicates that the measuring technique in this study is satisfactory.

Measurements of stature \& all parameters of patellae in this study revealed that males presented with significantly greater mean values than females $(\mathrm{P}<0.05)$ and this indicates the presence of significant sexual dimorphism in all Egyptian patellae measurements (Table 2).

There was a strong +ve significant correlation between stature \& the different measurements of patella in both sexes $(\mathrm{P}<0.05)$. In males, the higher correlation coefficient is exhibited by $\mathrm{MH}(\mathrm{r}$ $=0.933) \&$ the lowest is EFW $(r=0.689)$. In females, the higher correlation coefficient is with $\mathrm{MH}(\mathrm{r}=0.965)$, while the lowest is with IFW ( $\mathrm{r}=0.804)$ (Table 3).

When the six significant measurements entered the discriminant function analysis for sex identification, the percentage of accuracy was highest with usage of MT $(85.9 \%)$ by simple discriminant analysis (Table 4). The percentage of accuracy was $85.9 \%$ by mul-

Table 1. Concordance Correlation Coefficient of Reproducibility (Pc).

\begin{tabular}{|c|c|}
\hline Variables & Pc \\
\hline $\mathrm{MH}$ & 0.99 \\
\hline $\mathrm{MFH}$ & 0.98 \\
\hline $\mathrm{MFB}$ & 0.97 \\
\hline $\mathrm{EFW}$ & 0.97 \\
\hline $\mathrm{IFW}$ & 0.95 \\
\hline $\mathrm{MT}$ & 0.97 \\
\hline
\end{tabular}

MH: maximum height, MFH: maximum height of articulating surface, MFB: maximum facet breadth, EFW: external articulating facet width, IFW: internal articulating facet width, MT: maximum thickness.

Table 2. Descriptive Statistics of the Patella for Egyptian Population (in mm).

\begin{tabular}{|c|c|c|c|}
\hline & $\begin{array}{c}\text { Male } \\
(\mathrm{N}=120)\end{array}$ & $\begin{array}{c}\text { Female } \\
(\mathrm{N}=78)\end{array}$ & P value \\
\hline $\begin{array}{c}\text { Stature Range } \\
\text { Mean } \pm \text { SD }\end{array}$ & $\begin{array}{c}(160-176) \\
167.6 \pm 4.1\end{array}$ & $\begin{array}{c}(158-170) \\
164.31 \pm 3.29\end{array}$ & \\
\hline $\begin{array}{c}\text { MH Range } \\
\text { Mean } \pm \text { SD }\end{array}$ & $\begin{array}{c}(37.9-52) \\
42.27 \pm 3.75\end{array}$ & $\begin{array}{c}(36.4-43.1) \\
39.72 \pm 2.01\end{array}$ & $<0.001^{*}$ \\
\hline MFH Range & $(28.5-43)$ & $(27-40.8)$ & $0.012^{*}$ \\
Mean \pm SD & $32.72 \pm 4.11$ & $31.21 \pm 4.03$ & \\
\hline MFB Range & $(40-53.2)$ & $(37-44.5)$ & $<0.001^{*}$ \\
Mean \pm SD & $43.92 \pm 3.34$ & $40.95 \pm 2.31$ & \\
\hline EFW Range & $(21.4-28.5)$ & $(20-24.5)$ & $<0.001^{*}$ \\
Mean \pm SD & $24.31 \pm 1.76$ & $22.37 \pm 1.34$ & $<0.001^{*}$ \\
\hline IFW Range & $(18.6-25.3)$ & $(17.6-21)$ & $<$ \\
Mean \pm SD & $21.81 \pm 1.8$ & $19.48 \pm 1.22$ & $<0.001^{*}$ \\
\hline MT Range & $(16-23.4)$ & $(16.4-20.8)$ & $<$ \\
Mean \pm SD & $19.81 \pm 1.71$ & $17.91 \pm 1.44$ & \\
\hline
\end{tabular}

MH: maximum height, MFH: maximum height of articulating surface, MFB: maximum facet breadth, EFW: external articulating facet width, IFW: internal articulating facet width, MT: maximum thickness. S.D: standard deviation; * $\mathrm{P}$ value is significant when $\mathrm{P}<0.05$. 
Table 3. Correlation Between Stature and Different Patella Measurements in Both Sexes.

\begin{tabular}{|c|c|c|c|c|}
\hline \multirow{2}{*}{} & \multicolumn{2}{|c|}{ Male } & \multicolumn{2}{c|}{ Female } \\
\cline { 2 - 5 } & $\mathbf{r}$ & P value & r & P value \\
\hline MH & 0.933 & $<0.001^{*}$ & 0.965 & $<0.001^{*}$ \\
\hline MFH & 0.903 & $<0.001^{*}$ & 0.84 & $<0.001^{*}$ \\
\hline MFB & 0.899 & $<0.001^{*}$ & 0.942 & $<0.001^{*}$ \\
\hline EFW & 0.689 & $<0.001^{*}$ & 0.839 & $<0.001^{*}$ \\
\hline IFW & 0.704 & $<0.001^{*}$ & 0.804 & $<0.001^{*}$ \\
\hline MT & 0.915 & $<0.001^{*}$ & 0.874 & $<0.001^{*}$ \\
\hline
\end{tabular}

MH: maximum height, MFH: maximum height of articulating surface, MFB: maximum facet breadth, EFW: external articulating facet width, IFW: internal articulating facet width, MT: maximum thickness. $r$ : correlation coefficient "weak $(r=0-0.24)$, fair $(r=0.25-0.49)$, moderate $(\mathrm{r}=0.5-0.74)$, strong $(\mathrm{r}=0.75-1)$

*: significant difference at $\mathrm{p}$ value $<0.05$.

Table 4. Simple Discriminant Functional Analysis for Sex Determination.

\begin{tabular}{|c|c|c|c|c|c|c|c|c|}
\hline & \multirow{2}{*}{ Wilk's lambda } & \multirow{2}{*}{ P value } & \multirow{2}{*}{ Constant } & \multirow{2}{*}{ Coefficient } & \multirow{2}{*}{ Sectioning point } & \multicolumn{3}{|c|}{ Accuracy (\%) } \\
\cline { 6 - 9 } & & & & & & In males & In females & total \\
\hline MH & 0.867 & $<0.001^{*}$ & -12.949 & 0.314 & -0.169 & 80 & 48.7 & 67.7 \\
\hline MFH & 0.968 & $0.012^{*}$ & -7.872 & 0.245 & -0.079 & 100 & 19.2 & 68.2 \\
\hline MFB & 0.807 & $<0.001^{*}$ & -14.340 & 0.335 & -0.211 & 75 & 48.7 & 64.6 \\
\hline EFW & 0.741 & $<0.001 *$ & -14.591 & 0.620 & -0.255 & 75 & 48.7 & 64.6 \\
\hline IFW & 0.663 & $<0.001^{*}$ & -13.067 & 0.625 & -0.308 & 90 & 48.7 & 73.7 \\
\hline MT & 0.749 & $<0.001 *$ & -11.826 & 0.620 & -0.25 & 90 & 79.5 & 85.9 \\
\hline
\end{tabular}

MH: maximum height, MFH: maximum height of articulating surface, MFB: maximum facet breadth, EFW: external articulating facet width, IFW: internal articulating facet width, MT: maximum thickness. *: significant difference at p value $<0.05$

Discriminant score $=$ constant $+($ coefficient $\mathrm{x}$ measure $)$, If the discriminant score $>$ sectioning point $\rightarrow$ it means male

If the discriminant score $<$ sectioning point $\rightarrow$ it means female

Table 5. Multiple Discriminant Functional Analysis for Sex Determination.

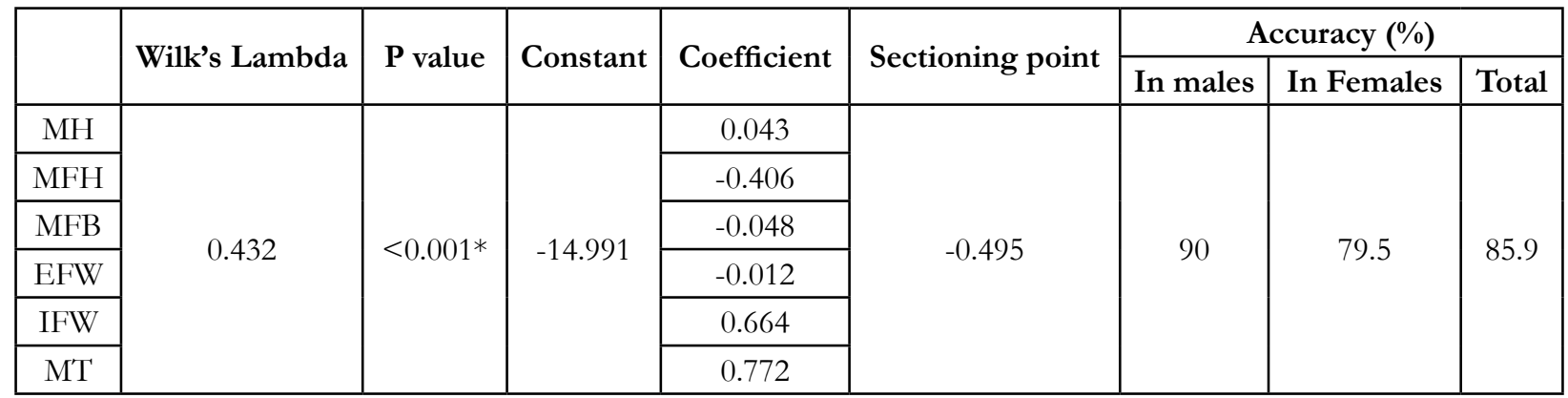

MH: maximum height, MFH: maximum height of articulating surface, MFB: maximum facet breadth, EFW: external articulating facet width, IFW: internal articulating facet width, MT: maximum thickness. ${ }^{*}$ : significant difference at p value $<0.05$

Discriminant score $=-14.991+(0.043 \times \mathrm{MH})+(-0.406 \times \mathrm{MFH})+(-0.048 \times \mathrm{MFB})+(-0.012 \times \mathrm{EFW})+(0.664 \times \mathrm{IFW})+(0.772 \times \mathrm{MT})$

If the discriminant score $>$ sectioning point $\rightarrow$ it means male

If the discriminant score $<$ sectioning point $\rightarrow$ it means female.

tiple discriminant function analysis, in which combination of all parameters was used (Table 5). By stepwise discriminant analysis, combination of (MFH, IFW \& MT) represented with percentage of accuracy of $85.9 \%$ (Table 6). Finally, discriminant score was calculated by constant + (coefficient $x$ measure) and then if the discriminant score $>$ sectioning point $\rightarrow$ it means male. And if the reverse occurs, it means female.
Linear regression analysis was used to estimate stature from patellar measurements. In males, by simple linear regression, the most accurate variable that can predict stature was $\mathrm{MH}(\mathrm{R}=0.933$ \& $\left.\mathrm{R}^{2}=0.871\right)$ followed by MT $\left(\mathrm{R}=0.915 \& \mathrm{R}^{2}=0.838\right)$ (Table 7). By multiple linear regression analysis, combination of all 6 measurements was used to predict stature $\left(R=0.972 \& R^{2}=0.945\right)$ 
(Table 8). In stepwise linear regression analysis, 3 models can be used to predict stature in males. The $1^{\text {st }}$ model is the combination of $(\mathrm{MH}, \mathrm{MT} \& \mathrm{MFB})\left(\mathrm{R}=0.972 \& \mathrm{R}^{2}=0.944\right)$. The 2 nd predictable model is the combination of $(\mathrm{MH} \& \mathrm{MT})\left(\mathrm{R}=0.964 \& \mathrm{R}^{2}=\right.$ 0.930). The last model is the use of $\mathrm{MH}$ alone $\left(\mathrm{R}=0.933 \& \mathrm{R}^{2}=\right.$ 0.871) (Table 9).

In females, the most predictable accurate variable in estimation of stature by simple linear regression is $\mathrm{MH}\left(\mathrm{R}=0.965 \& \mathrm{R}^{2}\right.$ $=0.931)$ followed by MFB $\left(\mathrm{R}=0.942 \& \mathrm{R}^{2}=0.887\right)$ (Table 7). By multiple regression analysis, combination of all measurements was used $\left(\mathrm{R}=0.981 \& \mathrm{R}^{2}=0.963\right)$ (Table 8). In stepwise linear regression analysis, 4 models were used to predict stature. 1 st model was the combination of (MH, MT, MFH \& MFB) $(\mathrm{R}=0.981 \&$ $\left.\mathrm{R}^{2}=0.962\right)$, while the $2 \mathrm{nd}$ model was the combination of $(\mathrm{MH}$, MT \& MFH $)\left(\mathrm{R}=0.980 \& \mathrm{R}^{2}=0.960\right)$. The $3 \mathrm{rd}$ model was the combination of (MH \& MT) $\left(\mathrm{R}=0.975 \& \mathrm{R}^{2}=0.950\right)$. The last model was $\mathrm{MH}$ alone $\left(\mathrm{R}=0.965 \& \mathrm{R}^{2}=0.931\right)$ (Table 9).

\section{Discussion}

Forensic anthropology represents the application of knowledge

Table 6. Stepwise Multiple Discriminant Functional Analysis for Sex Determination.

\begin{tabular}{|c|c|c|c|c|c|c|c|c|}
\hline & \multirow{2}{*}{ Wilk's lambda } & \multirow{2}{*}{$P$ value } & \multirow{2}{*}{ Constant } & \multirow{2}{*}{ Coefficient } & \multirow{2}{*}{ Sectioning Point } & \multicolumn{3}{|c|}{ Accuracy (\%) } \\
\hline & & & & & & In males & In females & Total \\
\hline MFH & \multirow{3}{*}{0.432} & \multirow{3}{*}{$<0.001 *$} & \multirow{3}{*}{-15.111} & -0.399 & \multirow{3}{*}{-0.495} & \multirow{3}{*}{90} & \multirow{3}{*}{79.5} & \multirow{3}{*}{85.9} \\
\hline IFW & & & & 0.648 & & & & \\
\hline MT & & & & 0.756 & & & & \\
\hline
\end{tabular}

MH: maximum height, MFH: maximum height of articulating surface, MFB: maximum facet breadth, EFW: external articulating facet width, IFW: internal articulating facet width, MT: maximum thickness. *: significant difference at p value $<0.05$

Discriminant score $=-15.111+(-0.399 \times \mathrm{MFH})+(0.648 \times \mathrm{IFW})+(0.756 \times \mathrm{MT})$

If the discriminant score $>$ sectioning point $\rightarrow$ it means male

If the discriminant score $<$ sectioning point $\rightarrow$ it means female

Table 7. Simple Linear Regression Analysis to Predict The person's Stature in Both Sexes.

\begin{tabular}{|c|c|c|c|c|c|c|c|}
\hline Model Male & B & SEM & $\mathbf{R}$ & $\mathbf{R} 2$ & SEE & P value & Regression equation \\
\hline $\begin{array}{c}\text { MH } \\
\text { Constant }\end{array}$ & $\begin{array}{c}1.02 \\
124.52\end{array}$ & $\begin{array}{l}0.03 \\
1.53\end{array}$ & 0.933 & 0.871 & 1.48 & $<0.001 *$ & $\mathrm{~S}=124.52+(1.02 \mathrm{x} \mathrm{MH})$ \\
\hline $\begin{array}{c}\text { MFH } \\
\text { Constant }\end{array}$ & $\begin{array}{c}0.9 \\
138.12 \\
\end{array}$ & $\begin{array}{c}0.03 \\
1.3 \\
\end{array}$ & 0.903 & 0.816 & 1.76 & $<0.001 *$ & $\mathrm{~S}=138.12+(0.9 \mathrm{x} \mathrm{MFH})$ \\
\hline $\begin{array}{c}\text { MFB } \\
\text { Constant }\end{array}$ & $\begin{array}{c}1.1 \\
119.14\end{array}$ & $\begin{array}{l}0.05 \\
2.17\end{array}$ & 0.899 & 0.809 & 1.8 & $<0.001 *$ & $\mathrm{~S}=119.14+(1.1 \mathrm{x} \mathrm{MFB})$ \\
\hline $\begin{array}{c}\text { EFW } \\
\text { Constant }\end{array}$ & $\begin{array}{c}1.6 \\
128.72\end{array}$ & $\begin{array}{l}0.15 \\
3.77\end{array}$ & 0.689 & 0.475 & 2.98 & $<0.001 *$ & $\mathrm{~S}=128.72+(1.6 \mathrm{x} \mathrm{EFW})$ \\
\hline $\begin{array}{c}\text { IFW } \\
\text { Constant }\end{array}$ & $\begin{array}{c}1.6 \\
132.64\end{array}$ & $\begin{array}{l}0.14 \\
3.26\end{array}$ & 0.704 & 0.495 & 2.92 & $<0.001 *$ & $S=132.64+(1.6 x$ IFW $)$ \\
\hline $\begin{array}{c}\text { MT } \\
\text { Constant }\end{array}$ & $\begin{array}{c}2.19 \\
124.13\end{array}$ & $\begin{array}{l}0.09 \\
1.76\end{array}$ & 0.915 & 0.838 & 1.66 & $<0.001^{*}$ & $\mathrm{~S}=124.13+(2.19 \mathrm{x}$ MT $)$ \\
\hline Female Model & B & SEM & $\mathbf{R}$ & $\mathbf{R} 2$ & SEE & $P$ value & Regression equation \\
\hline $\begin{array}{c}\mathrm{MH} \\
\text { Constant }\end{array}$ & $\begin{array}{c}1.58 \\
101.52\end{array}$ & $\begin{array}{l}0.05 \\
1.96\end{array}$ & 0.965 & 0.931 & 0.87 & $<0.001 *$ & $\mathrm{~S}=101.52+(1.58 \mathrm{x} \mathrm{MH})$ \\
\hline $\begin{array}{c}\text { MFH } \\
\text { Constant }\end{array}$ & $\begin{array}{c}0.68 \\
142.86 \\
\end{array}$ & $\begin{array}{c}0.05 \\
1.6 \\
\end{array}$ & 0.84 & 0.706 & 1.79 & $<0.001 *$ & $\mathrm{~S}=142.86+(0.68 \mathrm{x} \mathrm{MFH})$ \\
\hline $\begin{array}{c}\text { MFB } \\
\text { Constant }\end{array}$ & $\begin{array}{c}1.34 \\
109.27\end{array}$ & $\begin{array}{l}0.05 \\
2.25\end{array}$ & 0.942 & 0.887 & 1.11 & $<0.001 *$ & $\mathrm{~S}=109.27+(1.34 \mathrm{x} \mathrm{MFB})$ \\
\hline $\begin{array}{c}\text { EFW } \\
\text { Constant }\end{array}$ & $\begin{array}{c}2.06 \\
118.21\end{array}$ & $\begin{array}{l}0.15 \\
3.43\end{array}$ & 0.839 & 0.704 & 1.81 & $<0.001 *$ & $\mathrm{~S}=118.21+(2.06 \times \mathrm{EFW})$ \\
\hline $\begin{array}{c}\text { IFW } \\
\text { Constant }\end{array}$ & $\begin{array}{c}2.16 \\
122.08\end{array}$ & $\begin{array}{l}0.18 \\
3.59\end{array}$ & 0.804 & 0.646 & 1.97 & $<0.001 *$ & $\mathrm{~S}=122.08+(2.16 \mathrm{x} \mathrm{IFW})$ \\
\hline $\begin{array}{c}\text { MT } \\
\text { Constant }\end{array}$ & $\begin{array}{c}1.99 \\
128.58\end{array}$ & $\begin{array}{l}0.12 \\
2.28\end{array}$ & 0.874 & 0.764 & 1.61 & $<0.001 *$ & $\mathrm{~S}=128.58+(1.99 \times \mathrm{MT})$ \\
\hline
\end{tabular}

$\mathrm{B}=$ unstandardized coefficient, $\mathrm{R}=$ correlation coefficient, $\mathrm{R}^{2}=$ effect size, $\mathrm{SEE}=$ standard error of estimate, $\mathrm{SEM}=$ standard error of mean, *: significant difference at $\mathrm{p}$ value $<0.05 \mathrm{~S}$ : person's stature. 
Table 8. Multiple Linear Regression Analysis to Predict the Person's Stature in Both Sexes.

\begin{tabular}{|c|c|c|c|c|c|c|c|}
\hline Male Model & B & SEM & $\mathbf{R}$ & $\mathbf{R}^{2}$ & SEE & $P$ value & Regression Equation \\
\hline $\begin{array}{c}\text { MH } \\
\text { MFH } \\
\text { MFB } \\
\text { EFW } \\
\text { IFW } \\
\text { MT } \\
\text { Constant }\end{array}$ & $\begin{array}{c}1.07 \\
0.02 \\
-0.59 \\
-0.11 \\
0.02 \\
1.23 \\
125.49 \\
\end{array}$ & $\begin{array}{l}0.16 \\
0.07 \\
0.17 \\
0.11 \\
0.08 \\
0.11 \\
1.67 \\
\end{array}$ & 0.972 & 0.945 & 0.99 & $\begin{array}{c}<0.001^{*} \\
0.741 \\
0.001^{*} \\
0.346 \\
0.802 \\
<0.001^{*}\end{array}$ & $\begin{array}{c}\mathrm{S}=125.49 \\
+(1.07 \times \mathrm{MH}) \\
+(0.02 \times \mathrm{MFH}) \\
+(-0.59 \times \mathrm{MFB}) \\
+(-0.11 \times \mathrm{EFW}) \\
+(0.02 \times \mathrm{IFW}) \\
+(1.23 \times \mathrm{MT})\end{array}$ \\
\hline Female Model & B & SEM & $\mathbf{R}$ & $\mathbf{R}^{2}$ & SEE & $P$ value & Regression Equation \\
\hline $\begin{array}{c}\text { MH } \\
\mathrm{MFH} \\
\mathrm{MFB} \\
\mathrm{EFW} \\
\text { IFW } \\
\text { MT } \\
\text { Constant }\end{array}$ & $\begin{array}{c}1.14 \\
-0.11 \\
0.49 \\
0.17 \\
-0.51 \\
0.43 \\
100.16\end{array}$ & $\begin{array}{l}0.15 \\
0.06 \\
0.25 \\
0.17 \\
0.38 \\
0.24 \\
2.29\end{array}$ & 0.981 & 0.963 & 0.66 & $\begin{array}{c}<0.001 * \\
0.09 \\
0.059 \\
0.311 \\
0.194 \\
0.081\end{array}$ & $\begin{array}{c}\mathrm{S}=100.16 \\
+(1.14 \times \mathrm{MH}) \\
+(-0.11 \times \mathrm{MFH}) \\
+(0.49 \times \mathrm{MFB}) \\
+(0.17 \times \mathrm{EFW}) \\
+(-0.51 \times \mathrm{IFW}) \\
+(0.43 \times \mathrm{MT})\end{array}$ \\
\hline
\end{tabular}

$\mathrm{B}=$ unstandardized coefficient, $\mathrm{R}=$ correlation coefficient, $\mathrm{R}^{2}=$ effect size, $\mathrm{SEE}=$ standard error of estimate, $\mathrm{SEM}=$ standard error of mean; *: significant difference at $\mathrm{p}$ value $<0.05 \mathrm{~S}$ : person's stature.

Table 9. Multiple Stepwise Linear Regression Analysis to Predict the Person's Stature in Both Sexes.

\begin{tabular}{|c|c|c|c|c|c|c|c|}
\hline Male Model & B & SEM & $\mathbf{R}$ & $\mathbf{R}^{2}$ & SEE & $P$ value & Regression equation \\
\hline $\begin{array}{c}\text { MH } \\
\text { Constant }\end{array}$ & $\begin{array}{c}1.01 \\
124.52\end{array}$ & $\begin{array}{l}0.03 \\
1.53\end{array}$ & 0.933 & 0.871 & 1.48 & $<0.001 *$ & $\begin{array}{c}S=124.52 \\
+(1.01 \mathrm{MH})\end{array}$ \\
\hline $\begin{array}{c}\text { MH } \\
\text { MT } \\
\text { Constant }\end{array}$ & $\begin{array}{c}0.61 \\
1.07 \\
120.61 \\
\end{array}$ & $\begin{array}{c}0.04 \\
0.11 \\
1.2\end{array}$ & 0.964 & 0.930 & 1.09 & $\begin{array}{l}<0.001 * \\
<0.001 *\end{array}$ & $\begin{array}{c}S=120.61 \\
+(0.61 \times \mathrm{MH}) \\
+(1.07 \times \mathrm{MT})\end{array}$ \\
\hline $\begin{array}{c}\text { MH } \\
\text { MT } \\
\text { MFB } \\
\text { Constant } \\
\end{array}$ & $\begin{array}{c}1.16 \\
1.21 \\
-0.7 \\
125.12 \\
\end{array}$ & $\begin{array}{c}0.11 \\
0.1 \\
0.12 \\
1.35 \\
\end{array}$ & 0.972 & 0.944 & 0.98 & $\begin{array}{l}<0.001 * \\
<0.001 * \\
<0.001^{*}\end{array}$ & $\begin{array}{c}\mathrm{S}=125.12 \\
+(1.16 \times \mathrm{MH}) \\
+(1.21 \times \mathrm{MT}) \\
+(-0.7 \times \mathrm{MFB}) \\
\end{array}$ \\
\hline Female Model & B & SEM & $\mathbf{R}$ & $\mathbf{R}^{2}$ & SEE & $P$ value & Regression equation \\
\hline $\begin{array}{c}\text { MH } \\
\text { Constant }\end{array}$ & $\begin{array}{c}1.58 \\
101.52\end{array}$ & $\begin{array}{l}0.05 \\
1.96\end{array}$ & 0.965 & 0.931 & 0.87 & $<0.001 *$ & $\begin{array}{r}\mathrm{S}=101.52 \\
+(1.58 \mathrm{MH}) \\
\end{array}$ \\
\hline $\begin{array}{c}\text { MH } \\
\text { MT } \\
\text { Constant }\end{array}$ & $\begin{array}{c}1.25 \\
0.55 \\
104.64 \\
\end{array}$ & $\begin{array}{l}0.07 \\
0.11 \\
1.78 \\
\end{array}$ & 0.975 & 0.950 & 0.74 & $\begin{array}{l}<0.001 * \\
<0.001 *\end{array}$ & $\begin{array}{l}\mathrm{S}=104.64 \\
+(1.25 \mathrm{x} \mathrm{MH}) \\
+(0.55 \mathrm{x} M \mathrm{~T})\end{array}$ \\
\hline $\begin{array}{c}\text { MH } \\
\text { MT } \\
\text { MFH } \\
\text { Constant }\end{array}$ & $\begin{array}{c}1.46 \\
0.78 \\
0.19 \\
98.31\end{array}$ & $\begin{array}{l}0.08 \\
0.11 \\
0.05 \\
2.19\end{array}$ & 0.980 & 0.960 & 0.67 & $\begin{array}{l}<0.001^{*} \\
<0.001^{*} \\
<0.001^{*}\end{array}$ & $\begin{array}{c}\mathrm{S}=98.31 \\
+(1.46 \mathrm{x} \mathrm{MH}) \\
+(0.78 \mathrm{x} \mathrm{MT}) \\
+(0.19 \mathrm{x} \mathrm{MFH})\end{array}$ \\
\hline
\end{tabular}

$\mathrm{B}=$ unstandardized coefficient, $\mathrm{R}=$ correlation coefficient, $\mathrm{R}^{2}=$ effect size, $\mathrm{SEE}=$ standard error of estimate, $\mathrm{SEM}=$ standard error of mean, *: significant difference at $\mathrm{p}$ value $<0.05 \mathrm{~S}$ : person's stature.

and techniques that primarily concerned with the examination of material believed to be human to answer medicolegal questions including those related to identification. Forensic anthropology has a varied array of means and methods to identify the culprit as well as the victims in crime cases [11].

Stature and sex are of the most important and useful anthropometric parameters which determine the physical identity of an individual. Therefore, determination of sex \& stature from different body parts remains have obvious important in the identification of the criminals and victims [12]. There is a definite biologi- cal relationship of sex \& stature with all body parts such as head, trunk, vertebral column and extremities [13].

Patella is one of the largest sesamoid bones that is situated in front of knee joint in the quadriceps femoris tendon. It is a small compact bone that articulates with the distal anterior end of the femur. Patella is a solid element of human skeleton with no discernible morphological features for identification of sex and stature and no significant differences attributed to race [14].

Many forensic anthropologists used DNA in identification of 
Table 10. DFA Analysis Utilizing Patellar Parameters on Various Populations.

\begin{tabular}{|c|c|c|c|c|}
\hline Authors \& Year & DFA & Reported Parameter & Accuracy & Population \\
\hline O’Conner 1996 & $\mathrm{DA}$ & Height, width \&thickness & $\begin{array}{c}83 \% \text { in females \& } \\
78 \% \text { in males } \\
\end{array}$ & $\begin{array}{l}\text { Skeletal materials from Smith- } \\
\text { sonian institution in U.S.A }\end{array}$ \\
\hline \multirow{2}{*}{ Introna 1998} & UDA & Thickness & Right $83.8 \%$ & South Italian \\
\hline & MDA & Max. height & Right $83.8 \%$ & South Italian \\
\hline \multirow{2}{*}{$\begin{array}{l}\text { Bidmos et al., } \\
2005\end{array}$} & $\mathrm{UDA}$ & Max. height & $85 \%$ & South African Whites \\
\hline & MDA & All parameters & $85 \%$ & South African Whites \\
\hline \multirow{2}{*}{$\begin{array}{l}\text { Dayal \& Bidmos } \\
2005\end{array}$} & UDA & Max. width & $80 \%$ & South African blacks \\
\hline & $\mathrm{MDA}$ & $\begin{array}{l}\text { Max. width, Max. height } \\
\text { \& Max. thickness }\end{array}$ & $85 \%$ & South African blacks \\
\hline \multirow{2}{*}{ Kemkes 2005} & $\mathrm{UDA}$ & Max. height & $100 \%$ & \multirow{2}{*}{$\begin{array}{c}\text { Prehistoric Skeleton samples } \\
\text { from medieval period }\end{array}$} \\
\hline & MDA & Max. height & $100 \%$ & \\
\hline $\begin{array}{l}\text { Mahfouz et al., } \\
2007\end{array}$ & $\begin{array}{l}\text { Non linear } \\
\text { classification }\end{array}$ & All 6 parameters & $90.90 \%$ & \\
\hline Akhlagi et al 2010 & $\mathrm{DA}$ & $\begin{array}{l}\text { Max. width, Max. height } \\
\text { \& Max. thickness }\end{array}$ & $93 \%$ & Iranian population \\
\hline $\begin{array}{c}\text { Paolo Phoophalee } \\
\text { et al., } 2012 \\
\end{array}$ & $\mathrm{MDA}$ & All parameters & $90.50 \%$ & Northern Thai population \\
\hline $\begin{array}{c}\text { Kayalvizhi et al., } \\
2014 \\
\end{array}$ & MDA & $\begin{array}{c}\text { MH, MT, EFW, EFH, } \\
\text { MW }\end{array}$ & $80.60 \%$ & North Indian \\
\hline Kazuhiro 2008 & DFA & All parameters & $85 \%$ & Japanes \\
\hline \multirow{2}{*}{ Lis et al., 2014} & ANN model & \multirow{2}{*}{$\begin{array}{l}\text { Max. width, Max. height } \\
\text { \& Max. thickness }\end{array}$} & $96.10 \%$ & \multirow{2}{*}{ Malaysian population } \\
\hline & DFA & & $92.90 \%$ & \\
\hline
\end{tabular}

skeletal remains. But it has disadvantage that can't be extracted if skeleton is burned or damaged and so it cannot give data on some of the essential parameters of the biological profile. And so, measurements of skeletal remains either manually or by different radiological methods of can be helpful in these conditions [15].

The authors of this present study aware that patterns of sexual dimorphism vary among populations and the functions obtained could not have a similar accuracy if applied to different populations. So, the aim of this study was to use a non-invasive quick \& non-expensive method which is $3 \mathrm{D}$ CT for determination of sex \& stature from patella in a sample of Egyptian living subjects.

The results of this study revealed the presence of significant sexual dimoephism in all Egyptian patellae measurements. There was a strong positive significant correlation between stature \& the different parameters of patellae. B Univariate discriminant analysis, MT showed the highest percentage of accuracy (85.9\%) in sex identification. By stepwise discriminant analysis, combination of (MFH, IFW \& MT) represented with percentage of accuracy of $85.9 \%$. Also, by multiple discriminant analysis, the percentage of accuracy was $85.9 \%$.

There are a few studies about determination of sex from patella. The first one was made by Mc William and El Najjar in 1978 [16]. They used water displacement method to calculate the volume of patella. Most of the male patellae were more than $15 \mathrm{cc} \&$ the females were less than $11 \mathrm{cc}$. Another study was conducted by Gunn and Mc Williams [17] using volume of the bone. They reported the highest average accuracy for sex classification was $88 \%$ for Europeans.

In 1984, Rathbun and Rathbun [18] used the size and the shape of patella retrived with other human remains from a shark's stomach as supportive information for sex determination. They measured the patellae of 20 living white individuals and calculated a mean max. patellar height for males $(50.7 \pm 3.59)$ and females $(46.17 \pm$ $2.58)$ and a mean max. patellar width for males $(50.3 \pm 2.58)$ and females $(45.12 \pm 2.16)$. Their results were significantly higher than our results. The results of this present study revealed that the meam $\mathrm{MH}$ in males \& females were $(42.27 \pm 3.75 \& 39.72 \pm 2.01)$ respectively, and the mean MFB in males \& females were (43.92 \pm $3.34 \& 40.95 \pm 2.31)$ respectively.

Sex identification by subjecting some measurements of the patella was done by O, connor [19], Introna et al., [20], Bidomos [21], Dayal \& Bidmos [22], Kemkes [23], Mahfouz et al., [6], Akhlaghi et al., [3], Paolophoophalee et al., [24], Kayalvizhi et al [14], Kazuhiro [25], and Lis et al., [26] on different populations using discriminant function analysis of one or few or all of various measurements of patella without obtaining an equation as what we had done in this study. Their methods \& their accuracy for sex identification were tabulated (Table 10).

The study of Shang et al., [27] is the only study that used 3D CT to determine the presence of sexual dimorphism in all measurements of patella in living Chinese population. Although their study revealed the presence of significant sex difference but their study didn't include any discriminant analysis or percentage of accuracy.

We have found only one source that addressed sex determination from patella in Egyptian population. Anas et al., [28] identify sex from patellae that were taken from cadavers using sliding calipers graduated up to $0.5 \mathrm{~mm}$. They use the receiver operating characteristic (ROC) curve to detect the cut off values. Their study revealed that the maximum width represent a high percentage of accuracy $(93.55 \%)$. Combination of MT with the width of the 
lateral articular surface gave the highest rate of accuracy (95.2\%). Their study differed from our study in the method of determination of sex, statistical analysis \& results.

Kayalvizhi et al., [14] explained the differences in measurements of patella between males \& females due to the fact that males have a comparatively larger muscle build as compared to females and also the differences in body size. Also, the cortical bone in males has higher growth than in females.

The authors of this present study did not find any sources that addressed stature determination from the patella. In this current study the authors used linear regression analysis for estimation of stature from measurements of patella and derive an equation to identify stature. By simple linear regression analysis, the most accurate variable that can predict stature in both males $\&$ females was MH followed by MT in males \& MFB in females. By stepwise regression analysis, 3 models were used to predict stature in males which are (MH, MT \& and MFB) or (MH \& MT) and the last model was the use of $\mathrm{MH}$ only. Four models were used to predict stature in females, the first one was combination of $(\mathrm{MH}$, MT, MFH \& MFB), the 2nd model was (MH, MT \& MFH), the 3rd model (MH \& MT) and finally, MH was used alone to predict stature.

\section{Conclusion \& Recommendations}

This study has shown that measurements of patella are helpful in identification of sex and stature in Egyptians. Computed tomography scanning is a quick, non-destructive, inexpensive way to get morphometric measurements. The implementation of discriminant function \& linear regression analyses based on patellar morphometry offers a simple and a well studied approach for sex $\&$ stature identification. An important limitation of the technique is that, it cannot distinguish reliably between ethnic groups. So, it is recommended to do further analogous studies on patella and other skeletal remains from different populations.

Also, it is recommended to make future studies with modifications to this technique for MRI that may help to obtain more information from a large number of subjects from younger age. Optimization of scanning measuring techniques as well as increased numbers of samples can therefore be a subject of further investigations.

\section{Authors Contribution}

Shereen Abdelhakim was responsible for the idea of the research \& collection of data required for writing it, Osama A. Hassan was responsible for collection of references required for the research and writing parts of it, Inas Kamal Abdelazeem was responsible for collecting data for the research and writing it, Moustafa Abdel Kader was responsible for the practical method of the research anf MSCT examination.

\section{References}

[1]. Shah S, Patel P (2013) Sexing the human skull using the mastoid process. J Med Sci. 2(2): 75-78

[2]. Ogawa Y, Imaizumi K, Miyasaka S, Yoshino M (2013) Discriminant functions for sex estimation of modern Japanese skulls. J Forensic Leg Med. 20(4) : 234-238
[3]. Akhlaghi M, Sheikhazadi A, Ebrahimnia A, Hedayati M, Nazparvar B, et al., (2012) The value of radius bone in prediction of sex and height in the Iranian population. J Forensic Leg Med. 19(4): 219-222.

[4]. Torimitsu S, Makino Y, Saitoh H, Ishii N, Chiba F, et al., (2015) Stature estimation in Japanese cadavers based on scapular measurements using multidetector computed tomography. Int J Legal Med. 129(1): 211-8.

[5]. Yadav SK, Mandal BK, Karn A (2015) Determination of stature from ulnar length in Nepalese population. Eur J Forensic Sci. 2(1): 5-8.

[6]. Mahfouz M, Badawi A, Merkl B, Fatah EEA, Pritchard E, et al., (2007) Patella sex determination by $3 \mathrm{D}$ statistical shape models and nonlinear classifiers. Forensic Sci Int. 173(2-3): 161-170.

[7]. Borotikar BS, Sipprell WH, Wible EE, Sheehan FT (2012) A meth-odology to accurately quantify patellofemoral cartilage contactkinematics by combining 3D image shape registration and cine- PC MRI velocity data. Biomech. 45(6): 1117-1122.

[8]. Knuxmann R Anthropologie (1988) Handbuch der vergleichenden Biologie des Menschen. Band I/1. Stuttgart, Gustav Fischer.

[9]. Lin LI (1989) A concordance correlation coefficient to evaluate reproducibility. Biometrics 45(1): 225-68.

[10]. Cameron N (1984) The measurement of human growth, London: Croom Helm.

[11]. Blau S, Briggs CA (2011) The role of forensic anthropology in disaster victim identification (DVI). Forensic Sci Int. 205(1-3): 29-35.

[12]. Zeybek G, Ergur I, Demiroglu Z (2008) Stature and gender estimation using foot measurement. Forensic Sci Int. 181(1-3) : 54 e1-5.

[13]. Sheetal S, Surinder N (2014) Estimation of stature from different head and face measurements among male and female Jatavs of Delhi. IORS -JHSS. 19(9): 52-55.

[14]. Kayalvizhi I, Arora S, Dang B, Narayan RK (2015) Sex determination by applying discriminant functional analysis on patellar morphometry. Intl J sci Res. 4(11): 1511-1515.

[15]. Cattaneo C (2007) Forensic anthropology: developments of a classical discipline in the new millennium. Forensic Sci Int. 165(2-3) : 185-93.

[16]. El-Najjar MY, McWilliams KR (1978) Forensic anthropology: The structure, morphology, and variation of human bone and dentition. Charles $\mathrm{C}$. Thomas, Springfield, IL.

[17]. Gunn MC, McWilliams KR (1980) A method for estimating sex of the human skeleton from the volume of the patella, talus or calcaneus. Homo Gottingen. 31(3-4): 189-198.

[18]. Rathbun TA, Rathbun BC (1984) Human remains recovered from a shark's stomach in South Carolina. J Forensic Sci. 29(1): 269-276.

[19]. O'Connor WG (1996) The dimorphic sesamoid: differentiating the patella of females and males by height, width and thickness measurements. Master's thesis of arts in the department of Anthropology, University of South Carolina.

[20]. Introna F, Vella GD , Campobasso CP (1998) Sex determination by discriminant analysis of patella measurements. Forensic Sci Int. 95(1): 39-45.

[21]. Bidmos MA, Steinberg N, Kuykendall KI (2005) Patella measurements of South African Whites as sex assessors. Homo. 56(1): 69-74.

[22]. Dayal MR, Bidmos MA (2005) Discriminating sex in South African Blacks using patella dimensions. J Forensic Sci. 50(6) : 1294-7.

[23]. Kemkes Grottenthaler A (2005) Sex determination by discriminant analysis: an evaluation of the reliability of patella measurements. Forensic Sci Int. 147(2-3): 129-133.

[24]. Phoophalee P, Prasitwattanaseree S, Riengrojpitak S, Mahakkanukrauh P (2012) Sex determination by patella measurements in Thias, in proceedings of AGRC, Forensic Science Graduate program, Faculty of Science, Mahidol Univ., Bangkok, Thailand.

[25]. Kazuhiro S (2008) New method of the sex and age at death of an adult human skeleton from the patella. Bull. Natl Mus Nat Sci. 34: 43-51. https://www.kahaku.go.jp/research/publication/anthropology/download/34/ BNMNS_D3404.pdf

[26]. Lis A, Dewi N, Mohammed RA, Habibollah H (2016) Backpropagation neural network for sex determination from patella in forensic anthropology, Advances in computer science and its application. Lecture notes in electrical engenering. $279: 723-728$

[27]. Shang P, Zhang L, Hou Z, Bai X, Ye X, Xu Z (2014) Morphometric measurement of the patella on $3 \mathrm{D}$ model reconstructed from CT scan images for the southern Chinese population. Chin Med J 127(1): 96-101.

[28]. Anas M, Magdy M, Mona M, Emad S (1999) Identification of sex from patella in Egyptians. Mansoura J Forensic Med Clin Toxicol VII : 43-55. 